\title{
Automated Classification OF BANANA LEAF DISEASES USING AN OPTIMIZED CAPSUle NETWORK MODEL
}

\author{
Bolanle F. Oladejo and Oladejo Olajide Ademola \\ Department of Computer Science, University of Ibadan, Ibadan, Nigeria
}

\begin{abstract}
Plant disease detection and classification have undergone successful researches using Convolutional Neural Network (CNN); however, due to the intrinsic inability of max pooling layer in CNN, it fails to capture the pose, view and orientation of images. It also requires large training data and fails to learn the spatial relationship of the features in an object. Thus, Capsule Network (CapsNet) is a novel deep learn-ing model proposed to overcome the shortcomings of CNN. We developed an optimized Capsule Network model for classification problem using banana leaf diseases as a case study. The two dataset classes in- clude Bacterial Wilt and Black Sigatoka, with healthy leaves. The developed model adequately classified the banana bacterial wilt, black sigatoka and healthy leaves with a test accuracy of 95\%. Its outper- formed three variants of CNN architectures implemented (a trained CNN model from scratch, LeNet5 and ResNet50) with respect to rotation invariance.
\end{abstract}

\section{KEYWORDS}

Capsule Network, CNN, Activation function, Deep Learning, Precision Agriculture.

\section{INTRODUCTION}

In the recent years, there has been a significant upsurge in the use of computer vision techniques for image processing in precision agriculture. Banana (Musa Acuminata) is one of the most consumed staple food in Nigeria. It has great impart in food security and provide high intake of carbohydrates and calories for more than 70 million people in Sub-Saharan Africa [9]. However, there are some diseases affecting banana produce in Sub-Saharan Africa such as Banana Black Sigatoka, Banana Bacterial Wilt and Banana Streak etc. Farmers spends a lot of money to hire plant pathologist who manually checked the banana leaf in order to spot the diseases and provide a measure to control them. This process is prone to error and bias, hence the need for an automatic detection of these diseases using Artificial Intelligence techniques. Deep learning is a domain in Artificial Intelligence which automatically explore the features of data to perform a given tasks such as, computer vision, natural language processing etc.. It is a subject that has been explored in early days to solve vision problems such as object detection, image classification, disease diagnosis and classification etc. A state-of-the-art performance had been achieved using various deep learning techniques on a benchmark dataset such as ImageNet [8]. Convolutional Neural Network $(\mathrm{CNN})$ is one of the various artificial intelligence techniques which has been explored extensively in precision agriculture. However, given the unavailability of plant pathologist or agronomist in rural area, it is very challenging to get large amount of annotated diseased plant and healthy leaves dataset. The limited dataset issue causes CNN models to overfit which was why data augmentation techniques were employed to increase the size of dataset.

David C. Wyld et al. (Eds): ICAITA, CDKP, SAI, NCO, CMC, SOFT, MLT, AdNLP - 2020

pp. 119-130, 2020. CS \& IT - CSCP 2020

DOI: $10.5121 /$ csit.2020.100910 
The operations of CNN architecture does not consider the spatial relationship between the lower and higher level features of an object.. That is, they tend to lose information about feature locations, the spatial relationship of features and orientations of features in the input data. Hence, there is a need to design a deep learning model which could perform classification problem with better accuracy on small dataset. CNN architectures also fail to model the equivariance of an object and this is why a novel deep learning model was introduced by [18], referred to as Capsule Network (CapsNet) which can be seen as a group of neurons which models the hierachical relationships of features or objects. This object have different properties which includes the pose (position, size, orientation), deformation, velocity, albedo, hue, texture, among others.

CapsNet accepts input as a vector and it consists of Convolutional layer, Primary Capsule layer, DigitCaps layer, and the encoder-decoder. A capsule is designed as an activity vector of an input image whose overall length represents the probability or likelihood of the existence of an entity and its orientation represents its instantiation parameters. The capsule comprises a bunch of neurons stacked in capsules. The primary idea of Capsule Network is to replace the pooling algorithm of CNN with a Dynamic Routing Algorithm. Capsule Network is suitable for classification problem because of its ability to model the 3D structure of an object with the concept of inverse graphics [6]. CapsNet has achieved state-of-the-art performance on MNIST Handwritten Digit Recognition dataset by reducing the test error benchmark of CNN. However, with the current performance of capsule network, there is a room for improvement in order to augment its distinction on complex images (Three colour channel images). To the best of our knowledge, this is the first paper applying Capsule Network in Precision Agriculture. In order to augment the distinction of Capsule Network in terms of accuracy, ensuring faster convergence and reduc- ing the training time, in this paper, we propose an optimized version of CapsNet for Banana Leaf Disease Classification model.

The organization of this paper is as follows. Section (2) discusses the related works, and in section (3), we discuss the methodology of the optimized capsule network for banana leaf disease classification. Section (4) highlights the results and evaluation metric used for the designed model and the paper is concluded in Section (5).

\section{RELATED WORKS}

In this section, we review the related works in classification tasks using deep learning architectures. Generally, researchers have extensively explored the use of deep learning techniques for object recognition and image classification tasks $[3,4,13,15]$. Convolutional Neural Networks is a deep learning algorithm that has been used in recognition and classification problem and has achieved state of the art performances in image classification [10]. [17] developed the first CNN architecture named LeNet-5 for object detection and was evaluated with the handwritten digit MNIST dataset. The LeNet-5 architecture has 7 layers, which all contain trainable parame- ters (weights). It has two sets of convolutional, average pooling layers, flattening convolutional layer, two fully connected layers and the softmax classifier. It was designed before the advent of Graphics Processing Units (GPU) and one of the bottlenecks of this architecture is that it takes a longer time to train. In [2], an improved LeNet-5 architecture to classify two classes of banana leaf diseases was proposed. The limitations of the LeNet-5 architecture was opined by [14] and a deeper and wider version of the LeNet which was proposed won the ImageNet ILSVRC-2012 challenge with a test error rate of $15.3 \%$. AlexNet has been applied to various image classification task $[11,19]$. Other $\mathrm{CNN}$ architectures are also developed in order to reduce time complexity and number of trainable parameters.

It was noted in [3] that, the factors influencing the use of deep learning algorithms for plant disease detection and classification includes insufficient size and variety of annotated dataset which 
affect the performance and the generalizability of the deep learning models. However, a better way to overcome this shortcoming is to use transfer learning on a pre-trained network or perform data augmentation using Generative Adversarial Networks (GANs) [5, 13]. Another point raised by [3], is the symptom representation on plant leaves, Image background, multiplesimultaneous disorder, symptoms variation, disorders with similar symptoms and the covariate shift which is the phenomenon in which differences between the data used in training the model and the data on which the model is tested on results to low accuracies. The covariate shift prob- lem was observed by [18] where their model which has an accuracy of $99.5 \%$ drops drastically to $31 \%$ because the model was tested with a dataset from the cultivated field after being trained with a laboratory data.

In general, CNNs algorithms have been extensively explored in computer vision tasks but there are some shortcomings of CNNs that needs to be addressed. [6] gave some arguments about the limitations of convolutional neural networks which are: (i) CNN architectures are weak to affine transformation and do not consider much about the spatial relations of the features in the image,

(ii) The max-pooling layer in CNN tends to lose information while routing features from one layer to another, (iii) They fail to model the rotation invariance of an object. [18] proposed a Capsule Network with Dynamic Routing algorithm to overcome the drawbacks of CNN architecture. In [21], the capsule network architecture for gait recognition in which two operations were performed namely, matching local features at the bottom layer and matching mid-level features at the middle layer based on capsule network, was used, with the CASIA-B dataset and OUISIR Treadmill dataset B. Some researchers have also used capsule network on medical imaging for classification of diseases [1] and they have achieved better accuracies compared to the conventional CNN.

These above mention methods all utilized Image processing techniques to manipulate images and extract features for detection and classification task. However, the challenge with these methods is their inability to perform well on unconstrained imaging conditions like data gotten from the cultivated field. The dynamic routing by agreement of the capsule network makes it slower to train and, in a bid, to solve this challenge, [8] proposed a Matrix Capsule with ExpectationMaximization (EM) algorithm which uses a pose matrices. The routing process is similar to fitting a mixture of Gaussians using Expectation-Maximization, where each capsule in the in the lower layer is routed to a capsule with a similar vote in the higher layer. A novel deep learning approach for traffic sign classification using capsule network was also developed by [16]. Their model was trained and tested on German Traffic Sign Recognition Benchmark dataset (GTSRB) and they achieved a state-of-the-art accuracy of 97\%. Capsule networks have also been explored in medical imaging task and have achieved good accuracy compared to conven- tional convolutional neural network techniques. [1] used capsule network to classify brain tu- mour type on MRI images. They investigate the overfitting problem and also design a visualiza- tion paradigm to view the features learned from the MRI image dataset. Motivated by the performance of capsule network, we are proposing an optimized version of capsule network for banana leaf disease classification by improving the network in order to reduce the training time without a tradeoff for the accuracy of the network. To the best of our knowledge, this is the first work done in the problem domain. 
Table 1. Comparative literature review of existing model/framework for image processing using deep learning algorithm.

\begin{tabular}{lll}
\hline Paper & Description & Algorithm/Method \\
\hline$[2]$ & $\begin{array}{l}\text { A deep leaming based approach for banana leaf disease } \\
\text { classification. They Classify two (2) fungi banana leaf } \\
\text { diseases (Black Sigatoka and Banana Speckle) with a variant of }\end{array}$ & $\begin{array}{l}\text { Led ared to detect two types } \\
\text { of wanana leave disease. }\end{array}$ \\
& CNN based on LeNet5 architecture. &
\end{tabular}

[17]

[15]

[22]

[1]

\begin{abstract}
Using Deep Leaming for Image-Based Plant Disease Detection. They trained a convolutional neural network with 54,306 images of diseased and healthy plant leaves collected under controlled conditions to identify 14 crop species and 26 diseases.

A CNN model was trained to identify 14 crop species and 26 diseases.
\end{abstract}

Capsule Networks for Traffic Sign Classification was developed. They apply capsule network to traffic sign classification on German Traffic Sign Recognition Benchmark dataset (GTSRB). They achieved better performance and accuracy on the training and testing data compared to the conventional $\mathrm{CNN}$ architectures.

A novel three-charmel convolutional neural networks (TCCNN) model was developed by combining three color components of RGB for vegetable leaf disease recognition.

Brain tumour type classification using capsule network. They design a capsule network algorithm to investigate the overfitting problem with CNN algorithms on MRI images.
A Capsule Network model was developed to detect tra ffic signs.
CNN model was developed which makes used of the softmax layer for feature vector to classify the input veget-able leaf images.

Capsule Network for brain tumour classification

\section{Materials ANd Method}

The focus of this work is to design an Optimized Capsule Network model for banana leaf disease classification which will aid a faster detection and classification of banana leaf diseases. The following sections analyses the technique and approach used in implementing the Opti- mized Capsule Network model.

\subsection{Dataset}

The database was formed by collecting Banana Leaf disease and healthy images namely: Banana Bacterial Wilt disease (Xanthomonas wilt), Banana Black Sigatoka disease (Mycosphaerella fijiensis), and Healthy banana leaves. Three class labels were assigned to the collected dataset. We resized all the images collected to $256 \times 256$ pixels, preprocess and perform the training, validation and testing on the images. In all our experiments, we use the dataset with three colour channels (Red, Green, and Blue) without converting them to grayscale. $1000 \mathrm{im}$ ages were collected on the cultivated field across the three classes and the collected images were divided in the ratio of 80\%:20\% for training and testing respectively. The 20\% for testing was 
further divided in the ratio of 90\%:10\% for testing and validation respectively. Below is the sample image of the three classes of the dataset:

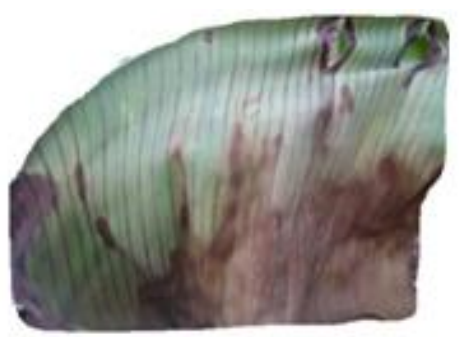

(a)

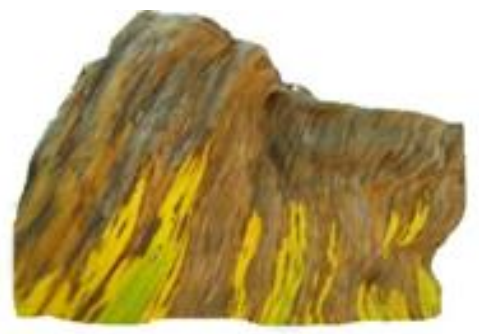

(b)

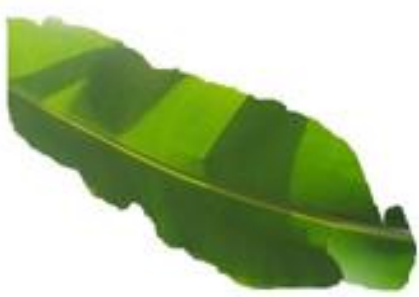

(c)

Figure 1. Sample of the banana leaf images collected. a. Banana Bacterial Wilt Disease image, b. Banana Black Sigatoka Disease image and c. Healthy leaf image.

\subsection{Data Preprocessing}

The dataset was collected and annotated with the aid of a plant pathologist. The annotated datasets were fed directly to the model without manual feature engineering to extract features. The data collected has no uniformity and in order to solve this problem, we applied data augmentation technique to the dataset in order to have a balanced data. Figure 2, Shows the flow of datasets collection, annotation and augmentation.

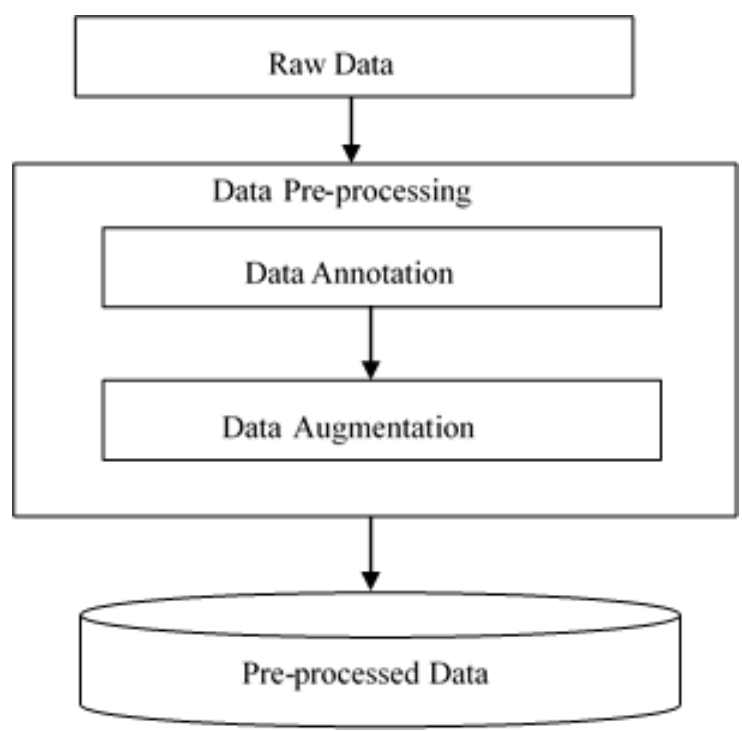

Figure 2. Schematic Diagram of Data Preprocessing.

\subsection{Development of an Optimized Capsule Network Model}

The Optimized Capsule Network (OCapsNet) model consists of the Input layer, Convolution layer, Primary capsule layer, DiseaseCaps layer and the fully connected layer. Figure 3 below shows OCapsNet architecture. The raw data of the input layer are down-sampled from $256 \times 256$ to $128 \times 128$ in order to reduce the number of parameters and in turn reduce the training time.

The first layer of our model is the input which consists of a three colour (Red, Green, and Blue) channel images of banana bacterial wilt disease, banana black Sigatoka leaf disease and healthy 
leaves with an input size of $128 \times 128 \times 3$. The second layer is the convolutional layer with Leaky-ReLU activation function to extract the features from the input data. In the convolutional layers, multiple kernels are used to extract the features from the banana leaf images and every kernel produces a feature map. In order to reduce dimensionality, we use convolution with a stride of 2 (The dimension is reduced by the factor of 2). The first convolutional layer has 256 channels, kernel size of $9 \times 9$, stride of 1 , and no padding while the second convolutional layer has 256 layers, kernel size of $9 \times 9$, stride of 2, and no padding. The third layer is the Primary Capsule (Primary Caps) layer in which the output of the convolutional layer is fed into the layer which contains multiple neurons at the same location in different feature maps grouped in capsules. The capsules in the primary capsule layer are connected with all the capsules in the Disease Caps layer where a dynamic routing-by-agreement algorithm is applied to check if the low- er level features learned is in agreement with the higher-level features. The dynamic routing-by agreement algorithm in figure 4 is a better alternative to the max-pooling operation in conventional Neural Networks (CNNs) as it tends to lose information while routing features from one layer to the other. The Disease Caps layer consists of three classes and the output is used to make a decision about the class of the input image. The number of neurons was reduced in the fully connected layer and an optimizer with momentum is used in order to have a faster network. The decoder consists of two fully connected layers with 512 and 1024 neurons respectively. We re- duced the number of the fully connected layer in the default architecture from three to two in other to reduce the training time of the network.

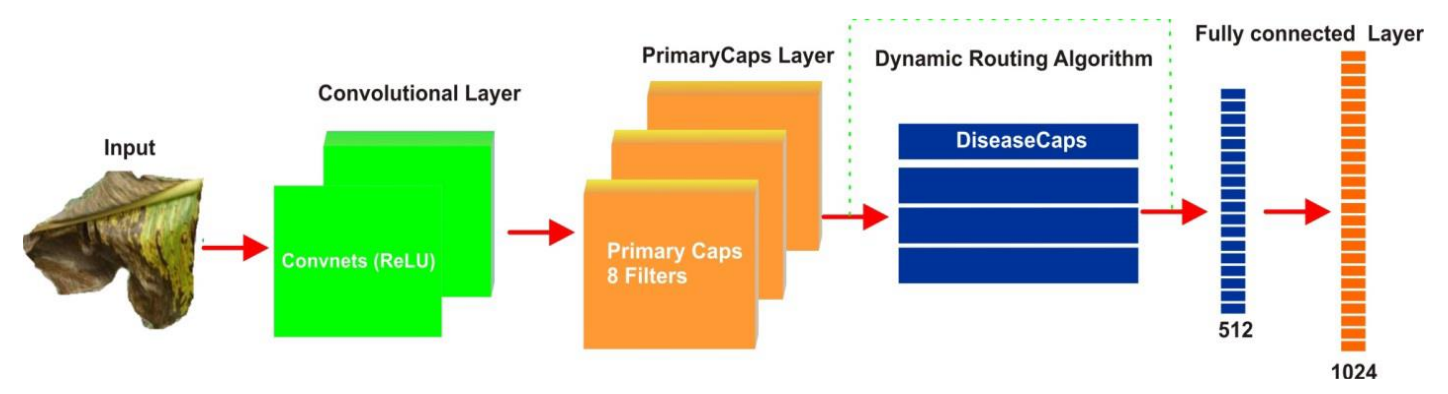

Figure 3. A schematic diagram of optimized capsule network model.

\subsubsection{Dynamic Routing Algorithm}

In capsule networks, the first convolution layer learns the basic features of an object and there is a need to route the features learned from the lower-level layer to the appropriate parent capsule representing the path-whole in the higher-level layers. The effective transfer of the features learned from one layer to another is made possible by the dynamic routing algorithm, the routing-by-agreement, which decides which entity from the lower level capsule, agrees with the output of the higher-level capsule through an iterative process. This process is done by a prediction or vote of a capsule $i$ in the lower layer $i$ for a capsule $j$ in higher layer which is produced by multiplying its pose by a weight matrix $W_{i j}$.

$$
\tilde{U}_{j / i}=W_{i j} u_{i}
$$

Where $\tilde{U}_{\mathrm{ji}}$ is a prediction vector gotten from the multiplication of the lower level vector $U_{i}$ and the weight matrix $\mathrm{W}_{\mathrm{ij}}$. The prediction is computed through a transformation matrix, and when multiple predictions from low-level capsules agree with the output of the high-level capsule, this gets activated. In order to get the weighted sum $\mathrm{Sj}$, the prediction vector is multiplied to the coupling coefficient $C_{\mathrm{ij}}$ which is determined by the routing-by-agreement algorithm. The routing algorithm calculates the agreement between the lower level vector and the higher level vector by doing a scalar product (.) of the prediction vector and the coupling coefficient. If the prediction 
vectors agree with each other, then the coupling coefficient will be higher which means, the two capsules are relevant to each other.

$$
\mathrm{Sj}=\sum \mathrm{i} \mathrm{Cij} \tilde{\mathrm{Uj}} \mid \mathrm{I}
$$

Where $\mathrm{C}_{\mathrm{ij}}$ is coupling coefficients that are calculated dynamically by the iterations of the dynamic routing. The coupling coefficients between one capsule and the others are sum to one and determined by a routing Soft Max whose initial $\log$ its $b_{i j}$ is the $\log$ prior probabilities that capsule in one layer should be coupled with the capsule in another layer. The agreement between these capsules is simply the scalar product of $a_{i j}=v_{j}$. $\tilde{U}_{j i}$.

$$
C_{i j}=\operatorname{softmax}\left(b_{i j)}=\frac{\exp \left(b_{i j}\right)}{\Sigma_{k} \exp \left(b_{i k}\right)}\right.
$$

The weighted sum $S_{j}$ is passed through a nonlinearity with a squash function in order to ensure that the vector length is between 0 and 1 . That is, if the length of the vector is 1 , there is a probability that a feature exists in the image.

$$
V_{j}=\frac{\left.|| S_{j}\right|^{2}}{1+\left.|| S_{j}\right|^{2}} \frac{S_{j}}{\|\left. S_{j}\right|^{2}}
$$

Algorithm 1. Dynamic Routing Algorithm procedure as described by [21].

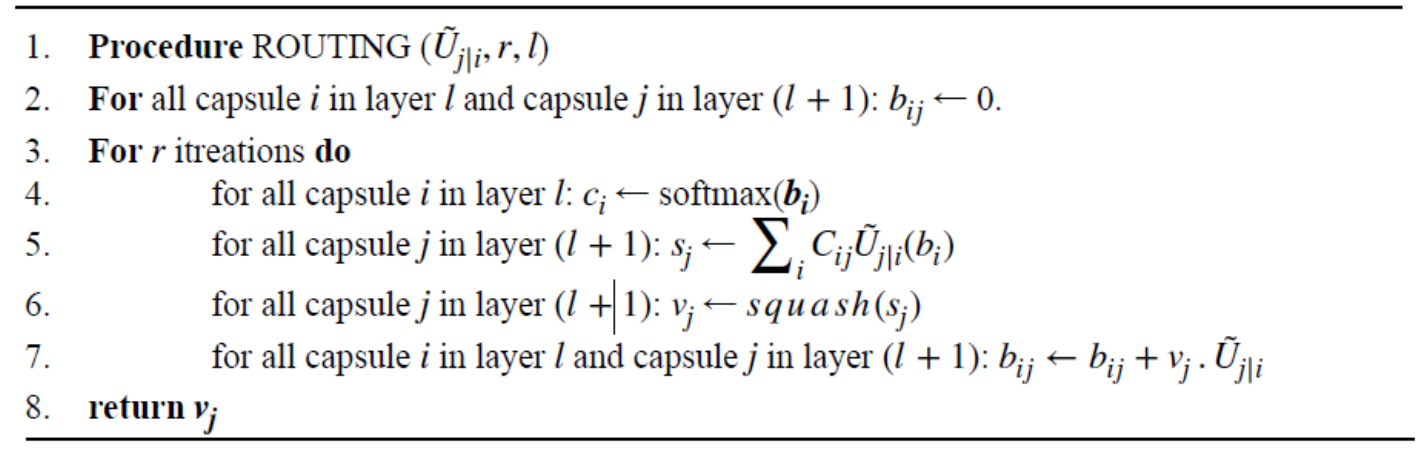

\subsubsection{Loss Function}

In order to train the network, two loses are employed which are, the margin loss and the reconstruction loss. The basic idea of the margin loss is to compute the error signal based on the length of the output vector of the DiseaseCaps. Hence, for the network to be able to segment highly overlapping objects, a loss is introduced in order to allow multiple object instances of different classes.

$$
L_{k}=T_{k} \max \left(o, m^{+}-|| v_{k} \mid\right)^{2}+\lambda\left(1-T_{k}\right) \max \left(0,|| v_{k}||-m^{-}\right)^{2}
$$

Where $T_{k}=1$ if and only if digit $\mathrm{k}$ is in the image, $m^{+}=0.9, m-=0.1$ and $\lambda=0.5$ is a downweighting parameter for absent digit classes that stops the initial learning from shrinking lengths of the activity vectors of all digit capsules. $v_{k}$ is the capsule that represents digit $k$ if a digit exists, 
$v_{k}$ will be high and the loss will be lower and if $v k$ is low, there would be a high loss. The total loss used for training is the sum of all margin losses for each digit defined by Loss $=\sum_{\mathrm{K}} L_{k}+$ $0.0005 R$.

\section{EXPERIMENT AND RESULT}

The model was trained, validated, and tested with the preprocessed dataset on a Google AI Cloud Service (Google Collaboratory) with the following configurations: Graphics Processing Unit (GPU) 1xTesla K80, having 2496 CUDA cores, computer 3.7, 12GB (11.439GB Usable) GDDR5 VRAM, CPU 1x single core hyper-threaded i.e. (1 core, 2 threads) Xeon Processors @ 2.3Ghz (No Turbo Boost), 45MB Cache, RAM 12.6 GB Available, Disk 320 GB Available. The model was implemented using Python programming language, TensorFlow as the backend and Keras library in Python 3.7. We ran the capsule network training 7 times by creating seven models, while we achieve the best result on the seventh model with an input size of $128 \times 128$.

\subsection{Evaluation Metric}

Accuracy evaluation metric was used in order to evaluate the proposed model. It is the proportion of the total number of predictions that were correctly classified i.e. the ratio of correctly predicted observation to the total observation.

$$
\begin{aligned}
& A C C=\frac{T P+T N}{P P} \\
& F P+F N+T P+T N
\end{aligned}
$$

Where True Positive rate (TP) and True Negative rate (TN) are correctly classified while False Positive (FP) and False Negative (FN) are correctly misclassified.

\subsection{Experimental Result}

The proposed capsule network model achieved a test accuracy of 0.953642 (95\%) using the collected datasets. Figure 4 and 5 below show the accuracy and loss graph of the proposed model. We also implemented a CNN model from scratch, LeNet5 and ResNet50 architecture and their results are reported in Table 2.

Table 2. Test Accuracy of Capsule Network, CNN, LeNet5 and Resnet50 architecture.

\begin{tabular}{|c|c|c|c|c|}
\hline \multicolumn{5}{|c|}{ Architectures } \\
\hline Models & Our model & CNN & LeNet5 & ResNet50 \\
\hline Accuracy & $95 \%$ & $89 \%$ & $82.5 \%$ & $96 \%$ \\
\hline
\end{tabular}




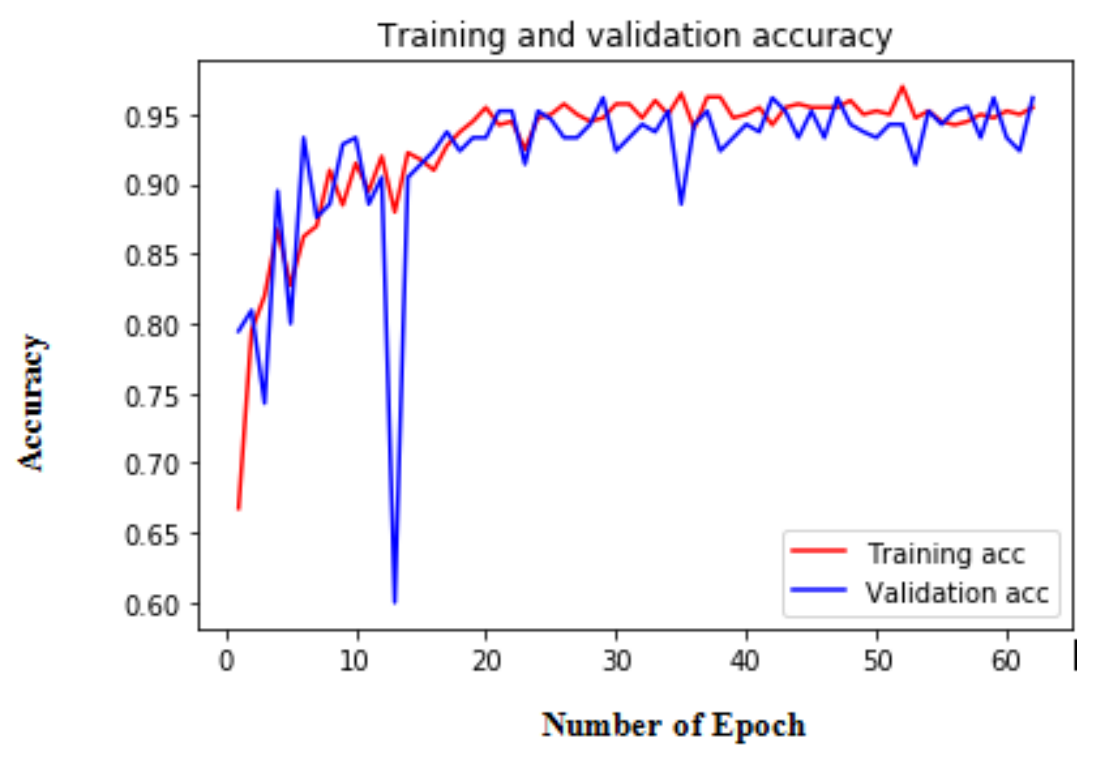

Figure 4. A graph showing the accuracy and validation of the optimized capsule network

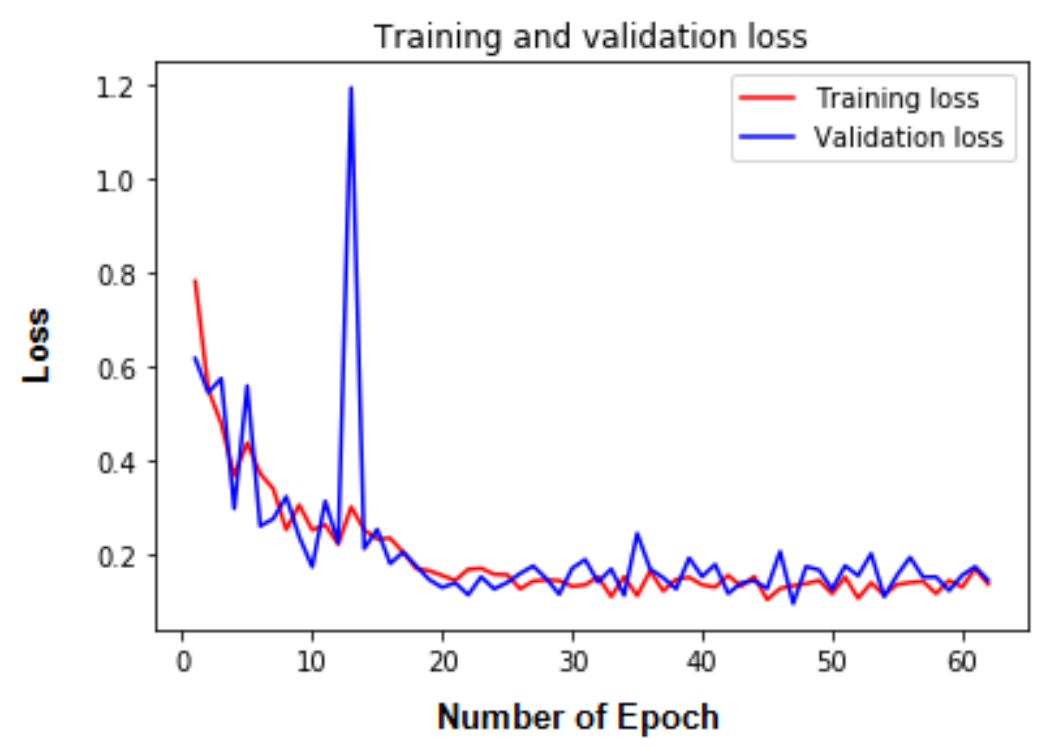

Figure 5. A graph showing the training and validation loss of the optimized network.

In order to compare our model with existing architectures, we implemented a CNN architecture from scratch, LeNet5 architecture and ResNet50 architecture using transfer learning. The CNN architecture achieved a test accuracy of $89 \%$, LeNet5 architecture archived a test accuracy of $82.5 \%$ while the ResNet50 architecture using transfer learning archived a test accuracy of $96 \%$. Table 2 above shows the results of the implemented architectures. We further rotate the test dataset in 30,60, and 90 degrees and used it to test the implemented models. Table 3 below shows the result obtained after the training dataset were rotated. Figure 6 further illustrates the visualization of the result of comparison among the different models. 
Table 3. Results of the Optimized Capsule Network, CNN, LeNet5 and ResNet 50 architecture when the test datasets were rotated to 30,60 and 90 degrees.

\begin{tabular}{|l|l|l|l|l|}
\hline Model & $\begin{array}{l}\text { Dataset without } \\
\text { rota- tion }\end{array}$ & $\begin{array}{l}\text { Test dataset with } \\
\text { 30 degrees } \\
\text { rotation }\end{array}$ & $\begin{array}{l}\text { Test dataset with } \\
\text { 60 degrees } \\
\text { rotation }\end{array}$ & $\begin{array}{l}\text { Test dataset with } \\
\text { 90 degrees } \\
\text { rotation }\end{array}$ \\
\hline $\begin{array}{l}\text { Capsule Net- } \\
\text { work }\end{array}$ & $95 \%$ & $93 \%$ & $95 \%$ & $95 \%$ \\
\hline CNN & $89 \%$ & $67.5 \%$ & $59 \%$ & $85.5 \%$ \\
\hline LeNet5 & $82.5 \%$ & $77 \%$ & $78 \%$ & $77 \%$ \\
\hline ResNet50 & $96 \%$ & $75 \%$ & $76 \%$ & $72 \%$ \\
\hline
\end{tabular}

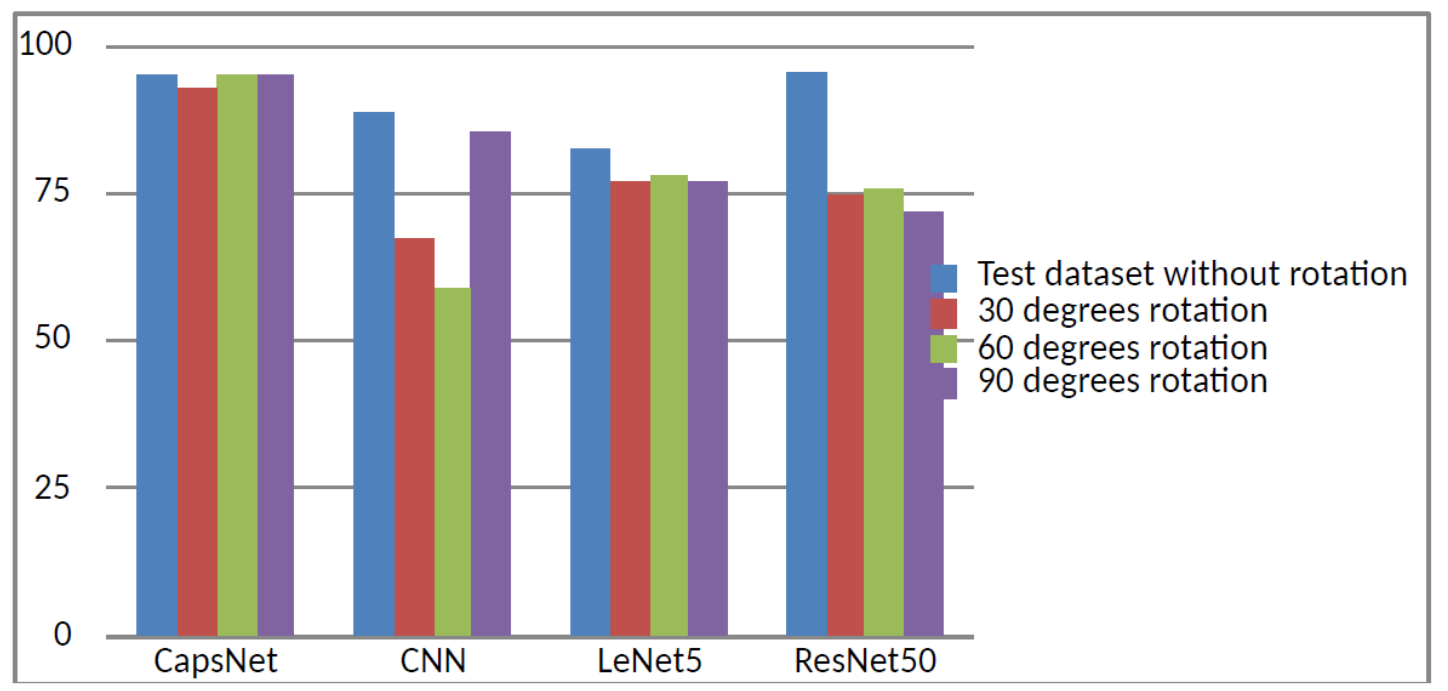

Figure 6. A bar chart showing the comparison of the implemented models.

\section{Conclusions}

In this paper, we proposed an optimized capsule network model for banana leaf disease classification. It is motivated by the newly proposed Capsule Network model which consists of the input layer, convolution layer, primary capsule layer and digitcaps layer. The network was optimized by fine-tuning the hyper parameters and using the Leaky ReLU activation function. The dataset used in this work contains two vari- ants of banana leaf diseases which are banana bacterial wilt disease (Xanthomonas wilt) and banana black sigatoka disease. We implemented the variants of $\mathrm{CNN}$ architecture (CNN trained from scratch, LenNet5 and ResNet50) in order to compare their performance with our model. The developed model outperformed other variants of CNN architectures implemented with respect to rotation invariance as seen in Table 3 above. However, the test dataset without rotation on ResNet50 architecture gives a better result than the proposed model. This work is limited to the two variants of banana (Musa Acuminata) leaf disease and future work includes building a robust capsule network model that can take care of diseases from various plant species. 


\section{REFERENCES}

[1] Afshar, P., Mohammadi, A., \& Plataniotis, K. N. (2018). Brain Tumor Type Classifica- tion via Capsule Networks. Proceedings - International Conference on Image Pro- cessing, ICIP, 3129-3133. Retrieved from: https://doi.org/10.1109/ICIP.2018.8451379

[2] Amara, J., Bouaziz, B. \& Algergawy, A., (2017). A DeepLearning-based Approach for Banana Leaf Diseases Classification. In: Mitschang, B., Nicklas, D., Leymann, F., Schöning, H., Herschel, M., Teubner, J., Härder, T., Kopp, O. \& Wieland, M. (Hrsg.), Datenbanksysteme für Business, Technologie und Web (BTW 2017) - Workshopband. Bonn: Gesellschaft für Informatik e.V. (S. 7988). Retrieved from: https://www. pdf- s.semanticscholar.org

[3] Barbedo, J. G. A. (2018). Factors influencing the use of deep learning for plant disease recognition. Biosystems Engineering, 172, 84-91. https://doi.org/10.1016/j.biosystem- seng.2018.0 5.013.

[4] Ferentinos, K. P. (2018). Deep learning models for plant disease detection and diagnos- is. Computers and Electronics in Agriculture, 145(September 2017), 311-318. https:// doi.org/10.1016/j.compag.2018.01.009.

[5] Goodfellow, I., Pouget-Abadie, J., Mirza, M., Xu, B., Warde-Farley, D., Ozair, S., \& Bengio, Y. (2014). Generative adversarial nets. In Advances in neural information pro- cessing systems (26722680).

[6] Hinton, G. E., Krizhevsky, A., \& Wang, S. D. (2011). Transforming autoencoders. In International Conference on Artificial Neural Networks 44-51. Springer, Berlin, Heidel- berg. https://doi.org/10.1007/978-3-642-21735-7_6.

[7] Hinton, G. E., Osindero, S., \& Teh, Y. W. (2006). A Fast Learning Algorithm for Deep Belief Nets (2016). Neural Computation, 18(7), 1527-1554. https://doi.org/10.1162/ neco.2006.18.7.1527.

[8] Hinton, G. E., Sabour, S., \& Frosst, N. (2018). Matrix capsules with EM routing. In In- ternational Conference on Learning Representations, 2018. https://openreview.net/fo- rum?id=HJWLfGWR b.

[9] Honfo, F. G., Tenkouano, A. \& Coulibaly, O. (2011). Banana and plantain-based foods consumption by children and mothers in Cameroon and Southern Nigeria: A comparative study. African Journal of Food Science Vol. 5(5), pp. 287 - 291, May 2011

[10] Huang, G., Liu, Z., Van Der Maaten, L., \& Weinberger, K. Q. (2017). Densely connec- ted convolutional networks. In Proceedings of the IEEE conference on computer vision and pattern recognition (pp. 4700-4708).

[11] Iandola, F. N., Han, S., Moskewicz, M. W., Ashraf, K., Dally, W. J., \& Keutzer, K. (2016). SqueezeNet: AlexNet-level accuracy with 50x fewer parameters and $<0.5 \mathrm{MB}$ model size. arXiv preprint arXiv: 1602.07360.

[12] Deng, J., Dong, W., Socher, R., Li, L., Kai, L. \& Fei-Fei, L. (2009). "ImageNet: A lar- gescale hierarchical image database," 2009 IEEE Conference on Computer Vision and Pattern Recognition, Miami, FL, 2009, pp. 248-255. doi: 10.1109/CVPR.2009.5206848.

[13] Kamilaris, A. \& Prenafeta, B. F. (2018). Deep Learning in Agriculture: A Survey. Com- puters and Electronics in Agriculture. 147. 10.1016/j.compag.2018.02.016.

[14] Krizhevsky, Alex \& Sutskever, Ilya \& E. Hinton, Geoffrey. (2012). ImageNet Classifi- cation with Deep Convolutional Neural Networks. Neural Information Processing Sys- tems. 25. DOI: $10.1145 / 3065386$.

[15] Simonyan, K. and Zisserman, A. (2015) Very deep convolutional networks for large- scale image recognition. In ICLR, 2015.

[16] Kumar, A. D., Engineering, C., Vidyapeetham, A. V., Engineering, C., Vidyapeetham, A. V., Parameswaran, L., \& Vidyapeetham, A. V. (n.d.) (2018). Novel Deep Learning Mo- del for Traffic Sign Detection Using Capsule Networks. arXiv preprint arXiv:1805.04424.

[17] Lecun, Y., Bottou, L., Bengio, Y., \& Ha, P. (1998). 00043771.Pdf, (November), $1-46$. https://doi.org/10.1109/5.726791.

[18] Mohanty, S. P., Hughes, D. P., \& Salathé, M. (2016). Using Deep Learning for Image- Based Plant Disease Detection. Frontiers in Plant Science, 7. https://doi.org/10.3389/ fpls.2016.01419.

[19] Sabour, S., Frosst, N., \& Hinton, G. E. (2017). Dynamic Routing Between Capsules, (Nips). https://doi.org/10.1371/journal.ponea.0035195

[20] Sivaranjini, S., \& Sujatha, C. M. (2019). "Deep learning based diagnosis of Parkinson's disease using convolutional neural network." Multimedia Tools and Applications (2019): 1- 13.

[21] Xu, Z., Lu, W., Zhang, Q., Yeung, Y., \& Chen, X. (2019). Gait recognition based on capsule network. Journal of Visual Communication and Image Representation, 59, 159- 167. 
[22] Zhang, S., Huang, W., \& Zhang, C. (2019). Three-channel convolutional neural net- works for vegetable leaf disease recognition. Cognitive Systems Research, 53,31-41. https://doi.org/10.1016/j.cogsys.2018.04.006.

\section{AUTHORS}

OLADEJO, Olajide Ademola, received his Master's Degree in Computer Science in year 2019 from the Department of Computer Science, University of Ibadan, Ibadan, Nigeria. He is working as a research coordinator at College of Medicine, University of Ibadan, Ibadan, Nigeria. He has published two papers in International Journals and one currently submitted at IEEE. His research interests include Artificial Intelligence, Computer Vision, Digital Health and Machine Learning.

Dr. Bolanle F. OLADEJO is a Senior lecturer at the department of Computer Science, University of Ibadan, Ibadan, Nigeria. She is a recipient of French Government scholarship for a co-supervised doctoral research. She holds a double $\mathrm{PhD}$ degree in Computer Science from Nancy 2 Universite (currently University of Lorraine), France and University of Ibadan. Her area of Specialization ranges from Knowledge pitalization/Management, Competitive/Business Intelligence, Software Engineering, and Health Informatics to Information Security. She has published over fourty papers from her research outputs in reputable conference

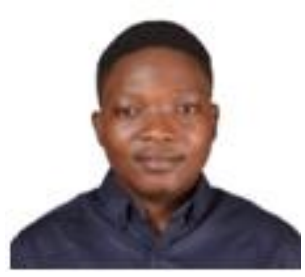
proceedings and peer-reviewed journals at both local and international levels. She leads a doctoral research team with bias in medical information visualization, decision support for security in terrorism and application of NLP and Knowledge Discovery techniques to collaborative knowledge sharing.

(C) 2020 By AIRCC Publishing Corporation. This article is published under the Creative Commons Attribution (CC BY) license. 